\title{
342 - The Dementia Early Stage Cognitive Aids New Trial (DESCANT) intervention: Goal Attainment Scaling
}

Helen Chester ${ }^{1}$, Rebecca Beresford ${ }^{2}$, Paul Clarkson ${ }^{2}$, Charlotte Entwistle ${ }^{2}$, Vincent Gillan ${ }^{2}$, Jane Hughes ${ }^{1}$, Martin Orrell ${ }^{1}$, Rosa Pitts ${ }^{2}$, lan Russell ${ }^{3}$, Eileen Symonds ${ }^{1}$, David Challis ${ }^{1}$, and Members of the HoSt-D (Home Support in Dementia) Programme Management Group

${ }^{1}$ Institute of Mental Health, School of Medicine, University of Nottingham, Nottingham, United Kingdom

${ }^{2}$ Social Care and Society, Division of Population Health, Health Services Research and Primary Care, School of Health Sciences, Faculty of Biology, Medicine and Health, University of Manchester, United Kingdom

${ }^{3}$ Swansea Trials Unit, Medical School, Swansea University, Swansea, United Kingdom

The DESCANT (Dementia Early Stage Cognitive Aids New Trial) intervention provided a personalised care package to improve the cognitive abilities, function and well-being of people with early-stage dementia and their carers by providing a range of memory aids, with training and support for use. This presentation will explore findings from a goal attainment scaling exercise undertaken within a multi-site pragmatic randomised trial, part of a NIHR-funded research programme 'Effective Home Support in Dementia Care: Components, Impacts and Costs of Tertiary Prevention.'

The aim was to describe the Goal Attainment Scaling (GAS) approach developed; investigate the types of goals identified by people with dementia and their carers and subsequent attainment; and explore the role of Dementia Support Practitioners (DSPs) in the process. This GAS exercise was designed by researchers, a clinical psychologist, a clinician and a DSP. Goal setting and attainment were conducted with the person with dementia and their carer and recorded by DSPs. Data were obtained from 117 intervention records and semi-structured interviews with five DSPs delivering the intervention across seven NHS Trusts in England and Wales. The GAS exercise was conducted as planned with goals and extent of involvement in the exercise tailored to individual participants and engagement was high. Demographic characteristics from the trial baseline dataset were analysed. Measures were created from intervention records to permit quantification and descriptive analysis. Interviews were professionally transcribed and subject to thematic analysis to identify salient themes.

A total of 293 goals were identified across the 117 participants. From these 17 goal types were distinguished across six domains: self-care; household tasks; daily occupation; orientation; communication; and well-being and safety. A measure of goal attainment appropriate to both the client group and a modest intervention was obtained. On average participants had evidenced some improvement regarding goals set. Qualitative findings suggested overall DSPs were positive about their experience of goal setting. Although several challenges were identified, if these were overcome, measuring goal attainment was generally viewed as straightforward. GAS can be used in the context of a psychosocial intervention for people with early-stage dementia to identify and measure attainment of personalised care goals. 